\title{
Performance of post-processing algorithms for rainfall intensity using measurements from tipping-bucket rain gauges
}

\author{
Mattia Stagnaro ${ }^{1,2}$, Matteo Colli ${ }^{1,2}$, Luca Giovanni Lanza ${ }^{1,2}$, and Pak Wai Chan ${ }^{3}$ \\ ${ }^{1}$ University of Genoa, Department of Civil, Chemical and Environmental Engineering, \\ Via Montallegro 1, 16145 Genoa, Italy \\ ${ }^{2}$ WMO/CIMO Lead Centre "Benedetto Castelli" on Precipitation Intensity, Genoa, Italy \\ ${ }^{3}$ Hong Kong Observatory, 134A Nathan Road, Hong Kong, China \\ Correspondence to: Mattia Stagnaro (mattia.stagnaro@unige.it)
}

Received: 29 July 2016 - Published in Atmos. Meas. Tech. Discuss.: 2 August 2016

Revised: 15 November 2016 - Accepted: 15 November 2016 - Published: 29 November 2016

\begin{abstract}
Eight rainfall events recorded from May to September 2013 at Hong Kong International Airport (HKIA) have been selected to investigate the performance of postprocessing algorithms used to calculate the rainfall intensity (RI) from tipping-bucket rain gauges (TBRGs). We assumed a drop-counter catching-type gauge as a working reference and compared rainfall intensity measurements with two calibrated TBRGs operated at a time resolution of $1 \mathrm{~min}$. The two TBRGs differ in their internal mechanics, one being a traditional single-layer dual-bucket assembly, while the other has two layers of buckets. The drop-counter gauge operates at a time resolution of $10 \mathrm{~s}$, while the time of tipping is recorded for the two TBRGs. The post-processing algorithms employed for the two TBRGs are based on the assumption that the tip volume is uniformly distributed over the inter-tip period. A series of data of an ideal TBRG is reconstructed using the virtual time of tipping derived from the drop-counter data. From the comparison between the ideal gauge and the measurements from the two real TBRGs, the performances of different post-processing and correction algorithms are statistically evaluated over the set of recorded rain events. The improvement obtained by adopting the inter-tip time algorithm in the calculation of the RI is confirmed. However, by comparing the performance of the real and ideal TBRGs, the beneficial effect of the inter-tip algorithm is shown to be relevant for the mid-low range $\left(6-50 \mathrm{~mm} \mathrm{~h}^{-1}\right)$ of rainfall intensity values (where the sampling errors prevail), while its role vanishes with increasing RI in the range where the mechanical errors prevail.
\end{abstract}

\section{Introduction}

Application-driven requirements of rainfall data (see e.g. Lanza and Stagi, 2008), the recommendations of international bodies such as the World Meteorological Organization (WMO, 2008), and new measurement quality standards issued at the national (UNI 11452:2012; BS 7843-3:2012) and international (CEN/TR 16469:2013) levels provide an increasingly demanding framework in terms of proven instrumental accuracy and reliability.

Following the effort led in the last decade by the WMO and aiming at quantifying the achievable accuracy of rainfall intensity measurements (Lanza and Vuerich, 2009), both users and manufacturers of precipitation gauges are developing strategies to reduce the uncertainty and to provide suitably documented performance evaluation of rainfall measurements.

Sound metrological procedures for the assessment of the uncertainty of meteorological measurements have recently been introduced within the framework of Europe-wide collaborative projects (Merlone et al., 2015) and therein extended to the measurement of liquid precipitation (see Santana et al., 2015). In this context, we use the term uncertainty in accordance with the International Vocabulary of Metrology (VIM) as the non-negative parameter characterizing the dispersion of the quantity values being attributed to a measurand (JCGM, 2012).

Besides the inherent instrumental factors (e.g. the systematic mechanical bias of tipping-bucket rain gauges or the dynamic response bias of weighing gauges), post-processing of 
the raw data to obtain accurate rain intensity records at a pre-determined temporal resolution is common practice. In the case of tipping-bucket rain gauges (TBRGs), dedicated post-processing algorithms must be employed to achieve sufficient accuracy and to minimize the impact of sampling errors and the discrete nature of the measurement.

Various algorithms have been proposed to this aim and discussed in the literature (Costello and Williams, 1991; Habib et al., 2001; Colli et al., 2013a, b). However, the operational practice of most users, including national weather services, still relies on the trivial counting of the number of tips occurring in the desired period. The number of tips counted in each 1 min time frame (the WMO recommended time resolution for rain intensity measurements) multiplied by the nominal volume of the bucket provides the 1 min rain intensity record.

This method (as already observed by Costello and Williams, 1991) results in a general underestimation of rain intensity figures and in a high level of uncertainty, due to the random nature of the number of tips per minute within any real-world, highly variable rainfall event. Moreover, the correction of systematic mechanical biases can not be optimized with this method since it would be applied to the averaged values only, and most tipping-bucket rain gauges show a non-linear correction curve after laboratory calibration (Lanza and Stagi, 2009).

We compare and discuss in this paper the performance of different post-processing algorithms employed in the calculation of the rainfall intensity from tipping-bucket rain gauges. Data recorded at a field test site by two TBRGs using different mechanical designs are used, and a catching-type drop-counting gauge is assumed as the working reference. The comparison aims to highlight the benefits of employing smart algorithms in post-processing of the raw data and their ability to improve the accuracy of rain intensity measurements obtained from TBRGs.

\section{Field site and instrumentation}

The Hong Kong Observatory performs rainfall measurements at the weather station of Hong Kong International Airport (HKIA). An Ogawa optical drop-counting rain gauge, model Osaka PC1122 (OSK), is available at the field site, providing rainfall measurements at the time resolution of $10 \mathrm{~s}$.

Based on the calibrated drop size volume (calculated in October 2013) of $63.93 \pm 0.56 \mathrm{~mm}^{3}$, the OSK dropcounter rain gauge is able to measure rainfall rates of up to $200 \mathrm{~mm} \mathrm{~h}^{-1}$ with a resolution of $5.21 \pm 0.04 \times 10^{-3} \mathrm{~mm}$ (Chan and Yeung, 2004; Colli et al., 2013a), thereby fulfilling the WMO (2008) accuracy requirements.

Due to the high accuracy and time resolution, we adopted the OSK drop-counter gauge as a working reference, and compared its measurements with co-located observations

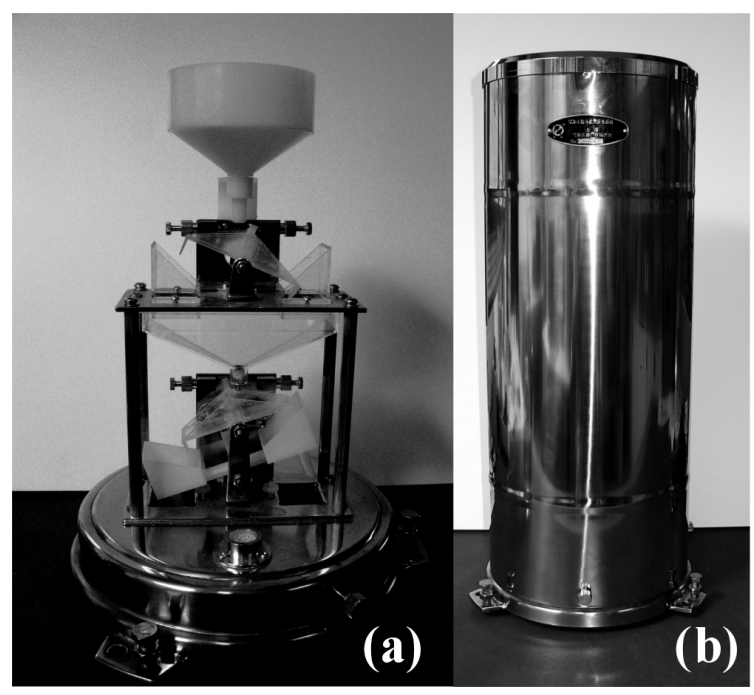

Figure 1. Shanghai SL3-1 (SL3): internal mechanism with a double layer of tipping buckets (a) and an external case (b).

performed by two TBRGs manufactured by Logotronic MRF-C (LGO) and Shanghai SL3-1 (SL3). The main characteristics of the instruments employed in this study are summarized in Table 1. Note, in particular, that the two TBRGs have the same nominal sensitivity (equal to $0.1 \mathrm{~mm}$ ) but employ different mechanical designs. The LGO is a traditional TBRG with a dual-compartment single-bucket assembly, while the SL3 gauge has two consecutive layers of dualcompartment buckets (see Fig. 1).

This is not a common solution for TBRGs, and the objective of the two layers of buckets employed seems to reside in the attempt to reduce the systematic mechanical bias, typical of traditional TBRGs. In a sense, this is a hardware type of correction similar to the use of a syphon or other mechanical solutions.

The three instruments are positioned in the western corner of the field test site of the Hong Kong Observatory (depicted in Fig. 2). The two TBRGs and the OSK drop counter are installed on the ground. The minimum distance from the main obstacle close to the instruments is about $18 \mathrm{~m}$. The positions of the SL3 (blue box), the LGO (red box) and the OSK (green box) are illustrated in Fig. 2; the distance between the SL3 and the OSK is about $5.8 \mathrm{~m}$, while the LGO is located about $2.1 \mathrm{~m}$ south of the OSK.

In order to correct the systematic mechanical errors of the two TBRGs, both of them were subjected to appropriate dynamic calibration in the laboratory. The dynamic calibration consists of providing the gauge with a sufficient number of equivalent rainfall intensities, using calibrated constant flow rates. By comparing the reference values with those measured by the rain gauge under test, the parameters of a suitable correction curve (usually a power law) are derived. The measurements from the two TBRGs were corrected before performing any comparison with the drop-counter time se- 
Table 1. Types of rain gauges employed for this comparison and their principal characteristics.

\begin{tabular}{llrrrr}
\hline Rain gauge & Measuring principles & $\begin{array}{r}\text { Resolution } \\
(\mathrm{mm})\end{array}$ & $\begin{array}{r}\text { Max. RI* } \\
\left(\mathrm{mmh}^{-1}\right)\end{array}$ & $\begin{array}{r}\text { Funnel diameter } \\
(\mathrm{mm})\end{array}$ & $\begin{array}{r}\text { Accuracy } \\
(\%)\end{array}$ \\
\hline Ogawa OSK PC1122 & Drop counter & $5.21 \times 10^{-3}$ & 200 & 127.0 & \pm 0.89 \\
Logotronic (LGO) & Tipping bucket & 0.1 & 200 & 252.3 & $\pm 2^{* *}$ \\
Shanghai (SL3) & Two-layer tipping bucket & 0.1 & 240 & 200.0 & $\pm 0.4^{* *}$ \\
\hline
\end{tabular}

* Maximum measured rainfall intensity as declared by the manufacturer

** Accuracy provided by the manufacturer.

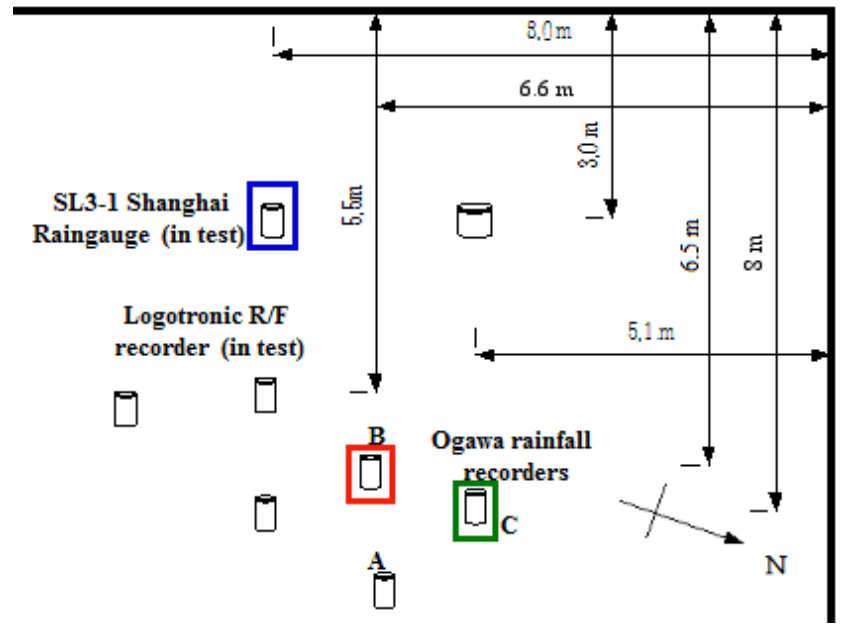

Figure 2. The western corner of the Hong Kong Observatory field test site where the SL3 (blue box), the LGO (red box) and the OSK (green box) are located. The distances of each instrument from the field site borders are also indicated.

ries and/or with the ideal TBRG data obtained from the reference.

In this work, the computation of statistical estimators and deviations between paired observations was performed with no reference to any ancillary data (wind speed and direction, air temperature and absolute pressure, etc.), although it is known that some of them (especially the wind) may actually affect the accuracy of the measurement.

The field data available for this study cover a 5-month period of observations from May to September 2013. Eight significant events in this period were selected based on the total rainfall depth, after checking that the reference rainfall intensity values were lower than the given factory limits for the instruments under test.

Table 2 reports a short description of the selected events, in terms of total rainfall depth $\left(h_{\text {tot }}\right)$, maximum rainfall intensity in $1 \min \left(I_{\max }\right)$ and event duration $(d)$.

Figure 3 shows a sample hyetograph of the raw data for the rain event that occurred on 22 May 2013. The 1 min reference rainfall intensity is depicted (shaded grey background) as calculated from the $10 \mathrm{~s}$ high-resolution data of the OSK drop counter, as well as the accumulated rainfall
Table 2. Total rainfall depth $\left(h_{\text {tot }}\right)$, maximum 1 min rainfall rate $\left(I_{\max }\right)$ and duration $(d)$ of selected events recorded by the Ogawa drop counter during the observation period.

\begin{tabular}{lrrr}
\hline Date & $\begin{array}{r}h_{\text {tot }} \\
(\mathrm{mm})\end{array}$ & $\begin{array}{r}I_{\max } \\
\left(\mathrm{mm} \mathrm{h}^{-1}\right)\end{array}$ & $d$ \\
\hline 22 May 2013 & 205 & 125 & 11h 27 min \\
25 May 2013 & 125 & 102 & 6h 56 min \\
24 June 2013 & 142 & 114 & 6h 19 min \\
24 July 2013 & 51 & 95 & 2h 54 min \\
25 July 2013 & 48 & 96 & 3h 10 min \\
4 September 2013 & 66 & 72 & 6h 44 min \\
5 September 2013 & 65 & 73 & 4h 53 min \\
23 September 2013 & 89 & 64 & 6h 9 min \\
\hline
\end{tabular}

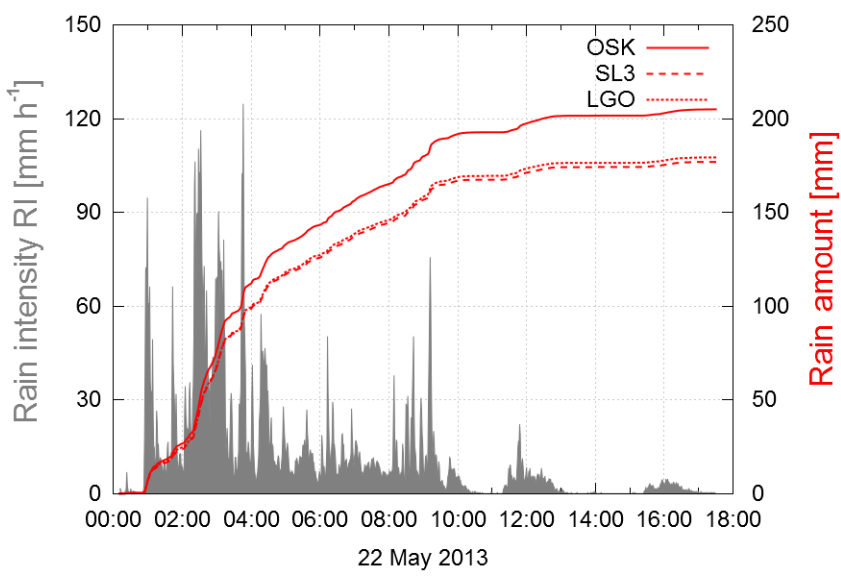

Figure 3. Reference rainfall intensity measured by the OSK drop counter (shaded grey background) and comparison of the accumulated reference (red continuous line) with the accumulated Logotronic (LGO) and Shanghai (SL3) measurements during the sample event of 22 May 2013.

for the OSK (solid red line) and the SL3 and LGO gauges (dashed and dotted line). The underestimation of the cumulative rain depth by the two TBRGs is evident from Fig. 3. The relative underestimation of the total depth, for this particular event, is equal to 13.6 and $12.5 \%$ for the SL3 and LGO rain gauges respectively. 


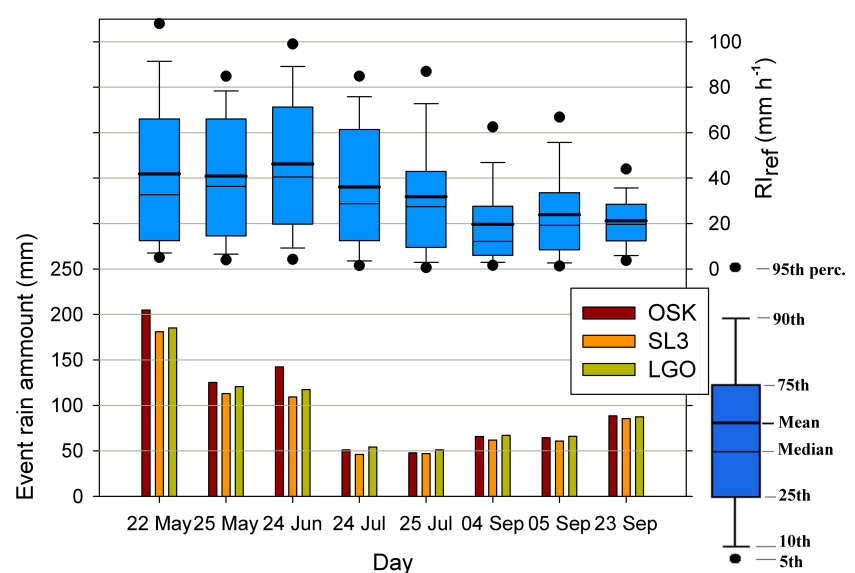

Figure 4. Box plot of the reference rainfall intensity for each event (top of the graph) and the corresponding daily rain amount (lower part of the graph) for the OSK drop counter (reference) and the two TBRGs (SL3 and LGO). The explanation of the symbols used in the boxplot representation is shown on the right-hand side of the graph.

Figure 4 reports a boxplot description of the set of events in terms of 1 min rainfall intensity distribution from the dropcounter reference OSK (upper part), together with the total accumulation for the OSK and the two TBRGs (lower part). Note that the two TBRGs show a larger underestimation of the total rain amount for events characterized by the highest values of the rainfall rate, in terms of both the mean and the extreme values, while the difference decreases for lower rainfall intensity (RI) events.

\section{Method}

We adopted the catching-type drop-counter gauge as the working reference for this work due to the high sensitivity of the measurement. Indeed, the instrument provides the number of generated drops with a time resolution of $10 \mathrm{~s}$. We first aggregated this information to obtain the $1 \mathrm{~min}$ reference rainfall intensity values $\left(\mathrm{RI}_{\mathrm{ref}}\right)$, for use in the overall assessment of the accuracy of the two involved TBRGs.

Both the SL3 and LGO rain gauges provide records of the time stamp of each tip. This feature allows one to use various algorithms to calculate the $1 \mathrm{~min}$ rainfall intensity values for the two investigated TBRGs.

The first, traditional and widely applied method to derive the 1 min rainfall intensity $\left(\mathrm{RI}_{\mathrm{raw}}\right)$ simply relies on the counting of the number of tips within each minute. The product of this number by the nominal volume of the bucket provides the rainfall amount in any single minute, and therefore the average rainfall intensity at such and any higher time resolution. The use of a suitable correction curve derived from laboratory calibration allows systematic mechanical errors to be accounted for as a function of the rainfall intensity. The traditional method assigns the whole volume of each bucket to the minute in which the tip occurs, even when part of the bucket is actually filled already in the previous minute, introducing significant counting errors in the calculation of the 1 min rainfall intensity $\mathrm{RI}_{\text {raw }}$. The uncertainty introduced by the tip-counting method also affects the efficacy of the calibration, since the correction applied to the volume of the bucket in each minute does not precisely derive from the actual rain intensity occurring in that minute.

The second method used to obtain the 1 min rainfall intensity values $\left(\mathrm{RI}_{\text {Ttip }}\right)$ employs the inter-tip time algorithm (see e.g. Costello and Williams, 1991; Colli et al., 2013a), which is based on the assumption that the nominal volume of each bucket is equally distributed over the inter-tip period. The calculation of the $\mathrm{RI}_{\text {Ttip }}$ for each minute accounts for the portion of the inter-tip period actually falling into that minute. In this way, the calibration is also the most effective since the correction applied to the volume of the bucket at the variable inter-tip scale is precisely the one corresponding to the measured rainfall intensity.

For both the TBRGs, the two values of the $1 \mathrm{~min}$ RI derived from the two post-processing algorithms described above (generally indicated in Eq. 1 as the measured rain intensity $\mathrm{RI}_{\mathrm{m}}$ ) were employed. We calculated the accuracy of rainfall intensity measurements in terms of percentage deviations $\left(e_{\text {rel }}\right)$ from the OSK drop-counter reference value $\left(\mathrm{RI}_{\text {ref }}\right)$ as follows:

$e_{\text {rel }}(\%)=\frac{\mathrm{RI}_{\mathrm{m}}-\mathrm{RI}_{\mathrm{ref}}}{\mathrm{RI}_{\mathrm{ref}}} \cdot 100$.

In addition, in order to compare the performance of postprocessing and correction algorithms for the TBRG measurements, we derived a virtual sequence of tips of an ideal tipping-bucket rain gauge $\left(\mathrm{TBR}_{\mathrm{i}}\right)$ from the high-resolution drop-counter (OSK) data. This simulates the behaviour of the best performing (optimal) TBRG, representing the maximum accuracy we can expect when using the tipping-bucket mechanics. The inter-tip and tip-counting algorithms were employed to derive the $1 \mathrm{~min}$ ideal rainfall intensity values $\left(\mathrm{RI}_{\mathrm{i}}\right.$ and $\mathrm{RI}_{\mathrm{i}(\text { raw })}$ respectively) and then the relative deviation $\left(e_{\text {rel }}\right)$ from the reference (OSK) as described in Eq. (1).

In order to assess the ability of the employed algorithm to describe the inner variability of the considered events and to capture their finer details, we calculated the correlation coefficients between all the derived time series and the reference ones. In particular, for each event, we calculated the RMSE error of paired deviations between the measured and ideal/reference RI signals.

In an effort to homogenize the dataset, we considered the standardized value of the generic rainfall intensity value $\left(\mathrm{RI}_{n}\right)$ obtained as follows:

$\mathrm{RI}_{n}=\frac{\left(\mathrm{RI}_{\mathrm{m}}-\mu_{\mathrm{ref}}\right)}{\sigma_{\mathrm{ref}}}$,

where the mean value $\mu_{\text {ref }}$ and the standard deviation $\sigma_{\text {ref }}$ employed in Eq. (2) to obtain the standardized values of rain- 


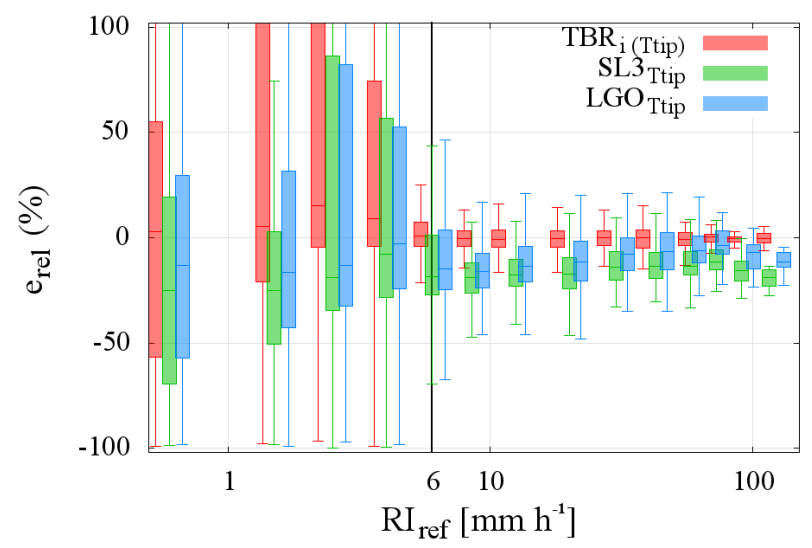

Figure 5. One-minute relative deviations $\left(e_{\text {rel }}\right)$ between the three TBRGs (including the ideal one) and the reference (OSK) when adopting the inter-tip post-processing algorithm (Ttip).

fall intensity $\left(\mathrm{RI}_{n}\right)$ for all the TBRGs are those derived from the OSK drop-counter reference rainfall rates. In this way, we obtained comparable results in terms of the standard deviation of the standardized RI time series for all the investigated rainfall events.

\section{Results}

We first evaluated the accuracy of the investigated RI algorithms using TBRG measurements by comparing their performance with the working reference. Figure 5 shows the relative deviations $\left(e_{\text {rel }}\right)$ from the drop-counter (OSK) reference for the three TBRGs (including the ideal virtual gauge). The reported box plots provide a synthesis of the results obtained by adopting the inter-tip algorithm, and are classified according to different ranges of $\mathrm{RI}_{\text {ref }}$.

Two different regions of this graph show different behaviours of the relative deviations calculated with the intertip approach. At low values of the RI, the relative deviations of all the TBRGs exhibit a large variability, whereas this scatter suddenly decreases just above the RI value of $6 \mathrm{mmh}^{-1}$ (highlighted in black) and then continues to decrease with increasing RI. This limit coincides with the sensitivity of the TBRG buckets (both the real and virtual ones); in fact, values of RI higher than $6 \mathrm{~mm} \mathrm{~h}^{-1}$ generate at least one tip per minute for TBRGs with a sensitivity of $0.1 \mathrm{~mm}$.

It emerges from the graph in Fig. 5 that the average deviation of both the LGO and SL3 gauges is always negative. In particular, the SL3 gauge shows an average underestimation that is larger than the LGO (except for one bin). This is coherent with the daily amounts shown in Fig. 4, where the cumulative rain depth of the SL3 gauge is lower than or equal to the value recorded by the LGO. Despite the fact that the SL3 rain gauge underestimates rainfall more than the LGO on average, the variability of the deviations from the reference for

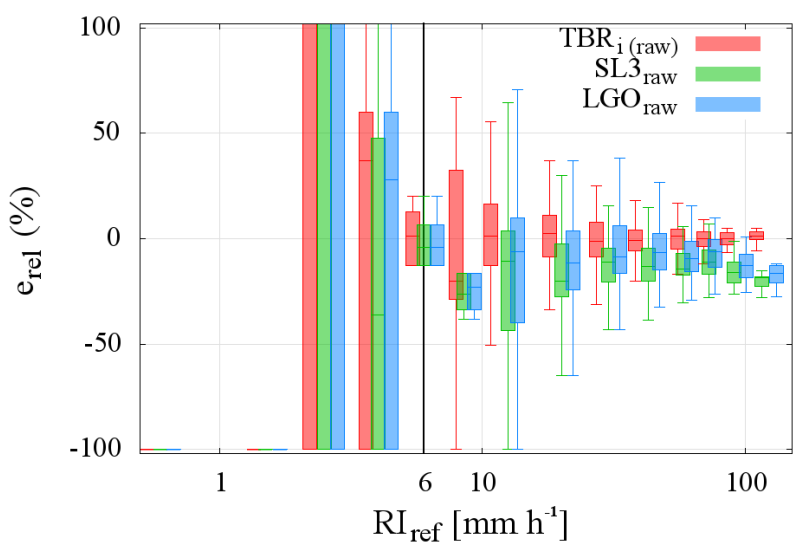

Figure 6. One-minute relative deviations $\left(e_{\text {rel }}\right)$ between the three TBRGs (including the ideal one) and the reference (OSK) when adopting the tip-counting algorithm (raw).

different values of RI is slightly reduced with respect to the LGO.

The behaviour of the ideal TBRG is clearly different, since it is not affected by instrumental mechanical errors (ideal mechanics), and the average value of $e_{\text {rel }}$ becomes close to zero immediately after the threshold value of the instrument sensitivity.

Note from Fig. 5 that the ideal TBRG shows large variability and an average value of the relative deviations that is comparable to the real TBRGs when the RI values are below $6 \mathrm{~mm} \mathrm{~h}^{-1}$. This means that the error caused by the aggregation time is relevant in this region when compared to the mechanical one.

As the rainfall rate increases, the variability of $e_{\text {rel }}$ considerably decreases, even if it does not vanish due to the sampling time of the OSK $(10 \mathrm{~s})$, whereas the average values are close to zero. The residual variability of $e_{\text {rel }}$ observed for the real TBRGs accounts for the propagation of the calibration uncertainty and other environmental factors (e.g. the windinduced and wetting effects).

Figure 6 describes the relative deviation $\left(e_{\text {rel }}\right)$ from the drop-counter OSK reference gauge of the measured value of RI from the three TBRGs adopting the rough tip-counting algorithm. Below the instrument sensitivity limit of $6 \mathrm{~mm} \mathrm{~h}^{-1}$, where less than one tip per minute occurs, the relative errors of all TBRGs are similar and show a very high scatter. Above this threshold value, the variability gradually decreases. Also, in this case the ideal TBRG shows an average value of deviation which is close to zero, while the SL3 and the LGO continue to maintain a mean value of $e_{\text {rel }}$ negative for all the RI classes. Close to the threshold value of $6 \mathrm{~mm} \mathrm{~h}^{-1}$, the variability of the relative error $\left(e_{\text {rel }}\right)$ decreases drastically for all three gauges, and the average value is the closest to zero. The residual variability of $\mathrm{e}_{\mathrm{rel}}$ observed for the real TBRGs when RI $>6 \mathrm{~mm} \mathrm{~h}^{-1}$ accounts for the residual tipping-bucket mechanical error after calibration and 


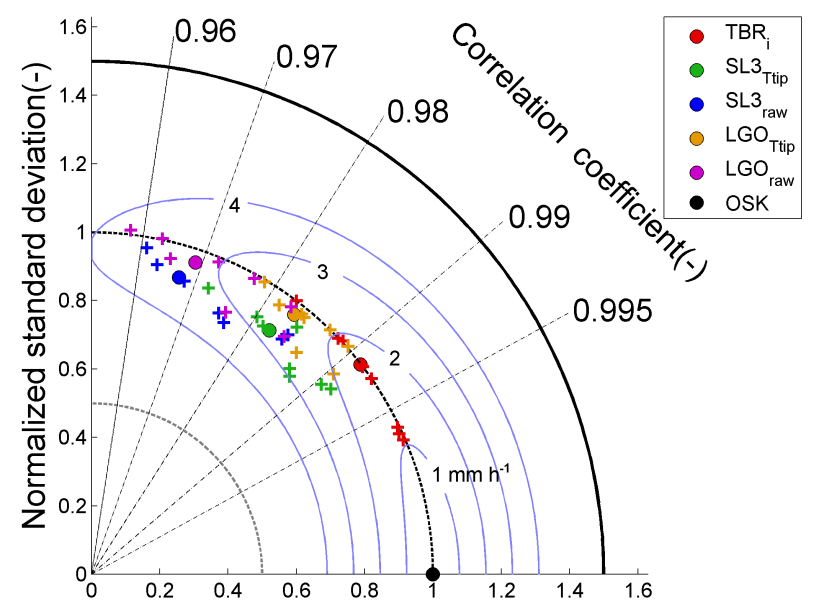

Figure 7. Taylor diagram representation of pattern statistics of the various RI series. The radial distance from the origin is proportional to the normalized standard deviation of the RI signal; the blue contour lines highlight the RMSE difference from the OSK reference (black dot) for each recorded event; the azimuthal position indicates the correlation coefficient between the RI signal and the reference. Crosses indicate the statistics of each single event, while the dots indicate the average values of the whole campaign (colours according to the legend).

other environmental factors (e.g. the wind-induced and wetting effects).

In Fig. 7 the Taylor diagram is reported (Taylor, 2001) to show the effect of the inter-tip algorithm in terms of the standard deviation of the standardized RI signal, the correlation coefficient between the TBRGs and the reference and its deviations from the reference. Considering the standard deviation of the RI signal for the real TBRGs, note that the two algorithms used to compute the RI values provide comparable results (approximately equal to 0.9). Since the ideal TBRG directly derives from the reference, the standardized standard deviation is the closest to unity.

In the same figure, the benefit of using the inter-tip time algorithm instead of the tip counting is evident: the correlation coefficients of the two real TBRGs increase using the former one for both TBRGs. Therefore, the beneficial effect in terms of deviations of the measured RI from the reference is highlighted. In fact, note the reduction of the RMSE difference from the reference, approaching the value of the ideal TBRG. This reduction can be quantified in about $1 \mathrm{~mm} \mathrm{~h}^{-1}$.

Also, the RI time series of the ideal TBRG shows a normalized standard deviation approximately equal to 1 , that is, the same as the reference, and a mean correlation coefficient greater than 0.99. However, the average value of the RMSE between the synthetic TBRG and the reference continues to show a relevant value slightly below $2 \mathrm{~mm} \mathrm{~h}^{-1}$, which does not decrease further below $1 \mathrm{mmh}^{-1}$ in all the considered events.

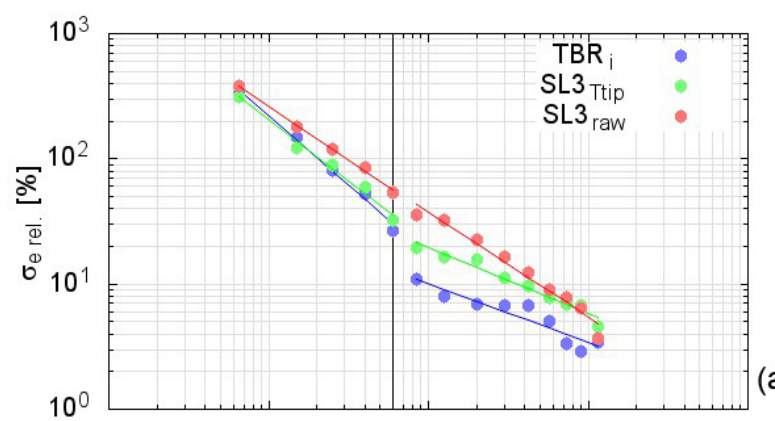

(a)
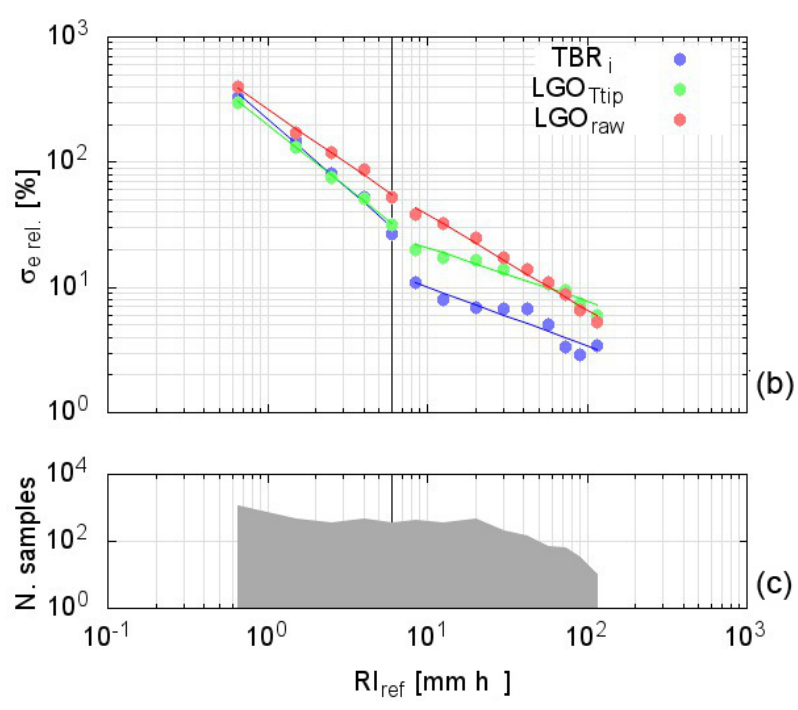

Figure 8. Standard deviation of the relative error for the ideal and investigated TBRGs when adopting the inter-tip time algorithm (Ttip) and the tip-counting method (raw) with respect to the ideal gauge for both the SL3 (a) ad LGO (b) instruments. In panel (c), the sample size for each RI class is reported.

In order to evaluate the effectiveness of the postprocessing algorithms on the accuracy of the measurements over different ranges of rainfall intensity, we plotted the standard deviation of the relative error $\left(e_{\text {rel }}\right)$ for different classes of RI. Figure 8 reports the results of this analysis.

It is evident from the graph that the raw counting of the number of tips results, for both the investigated TBRGs, in a continuous trend of linear (in a $\log -\log$ scale) reduction of the error variance with increasing RI. This reflects the fact that the random attribution of one tip to the wrong minute does not strongly affect the derived RI since the number of tips per minute is relatively high.

At very low values of RI, there is little difference between the results obtained by employing the simple counting of tips or the inter-tip algorithm, with respect to the ideal TBRG. By increasing the RI, but still below the threshold intensity corresponding to the sensitivity of the gauges, the effectiveness of the inter-tip time algorithm is relevant and results are very near to the ideal gauge. This effectiveness decreases beyond the sensitivity value and, when the RI increases beyond 50- 
$60 \mathrm{~mm} \mathrm{~h}^{-1}$, the difference with respect to the counting of tips becomes negligible. In this range, the LGO performs slightly better than the SL3 when the inter-tip time algorithm is used.

\section{Conclusions}

The raw data recorded during a dedicated monitoring campaign have been analysed using two different post-processing algorithms to calculate $1 \mathrm{~min}$ RI series. The results allow one to compare the performance of the inter-tip time algorithm with the more common tip-counting method when using two different types of TBRGs. The field reference chosen for this comparison is a catching-type, optical drop counter that, although calibrated in the laboratory, is still subject to unknown uncertainties in field operation (wind, wetting, splashing, etc.). Notwithstanding this residual uncertainty, comparison of the two gauges with a virtual TBRG obtained from the reference measurements was able to show relevant differences in the calculated 1 min rainfall intensity and the relationship of such differences with the rainfall rate itself.

In particular, the main benefit of adopting the inter-tip time method as a post-processing algorithm to calculate rainfall intensity from the raw data resides in a better representation of the inner variability of rainfall events. The measured RI series shows an improved correlation coefficient and a lower RMSE with respect to the reference, closely approaching the performance of an ideal TBRG, which is not affected by mechanical biases.

In terms of accuracy, the inter-tip time algorithm contributes its greater beneficial effects in the range of low to mid RI values. In the very low RI range, below the threshold value of $6 \mathrm{~mm} \mathrm{~h}^{-1}$ (corresponding to the sensitivity of the involved instrument and a typical value for an operational TBRG), the performance of the inter-tip time method in terms of the statistical amplitude of the deviations from the reference of the calculated RI are comparable to an ideal TBRG. In this range, indeed, the time lag between consecutive tips exceeds the time resolution of the measurement, and the sampling error represents the main source of uncertainty.

Beyond that threshold value, a step change is observed since at least one tip per minute is recorded when $\mathrm{RI}>6 \mathrm{mmh}^{-1}$. The inter-tip time algorithm is still better than the tip-counting method in this range, up to about $50 \mathrm{~mm} \mathrm{~h}^{-1}$, although the performance is no longer comparable to that of the ideal gauge. Mechanical errors become prevalent here, so that deviations from the ideal gauge result from the residual uncertainty of the calibration process. At the highest RI values the benefits of the inter-tip time algorithm vanish due to the large number of tips per minutes recorded in this range, and the performances of the two post-processing algorithms become comparable, though both of them perform worse than the ideal TBRG.

\section{Data availability}

The data presented in this paper are available on request from the corresponding author.

Acknowledgements. This research is supported through funding from the European Metrology Research Programme - project MeteoMet 2 (ENV58-REG3).

Edited by: G. Vulpiani

Reviewed by: R. Uijlenhoet and three anonymous referees

\section{References}

BS 7843-3:2012: Acquisition and management of meteorological precipitation data from a gauge network, Standard, British Standards Institution, 2012.

CEN/TR 16469:2013: Hydrometry - Measurement of the rainfall intensity (liquid precipitation): requirements, calibration methods and field measurements, Technical Report, European Committee for Standardization, 2013.

Chan, P. W. and Yeung, C. L.: A Study of Drop-counting Rain Gauges, Hong Kong Observatory, Technical Note (Local), No. 79, 14 pp., 2004.

Colli, M., Lanza, L. G., and Chan, P. W.: Co-located tipping-bucket and optical drop counter RI measurements and a simulated correction algorithm, Atmos. Res., 119, 3-12, $2013 \mathrm{a}$.

Colli, M., Lanza, L. G., and La Barbera, P.: Performance of a weighing rain gauge under laboratory simulated time-varying reference rainfall rates, Atmos. Res., 131, 3-12, 2013b.

Costello, T. A. and Williams, H. J.: Short duration rainfall intensity measured using calibrated time-of-tip data from a tipping bucket raingage, Agr. Forest Meteorol., 57, 147-155, 1991.

Habib, E., Krajewski, W. F., and Kruger, A.: Sampling Errors of Tipping-Bucket Rain Gauge Measurements, J. Hydrol. Eng., 6, 159-166, 2001.

JCGM: International vocabulary of metrology - Basic and general concept and associated terms (VIM), Joint Committee for Guidelines in Metrology, 3rd edn., 2012.

Lanza, L. G. and Stagi, L.: Certified accuracy of rainfall data as a standard requirement in scientific investigations, Adv. Geosci., 16, 43-48, doi:10.5194/adgeo-16-43-2008, 2008.

Lanza, L. G. and Stagi, L.: High resolution performance of catching type rain gauges from the laboratory phase of the WMO Field Intercomparison of Rain Intensity Gauges, Atmos. Res., 94, 555563, 2009.

Lanza, L. G. and Vuerich, E.: The WMO field intercomparison of rain intensity gauges, Atmos. Res., 94, 534-543, 2009.

Merlone, A., Lopardo, G., Sanna, F., Bell, S., Benyon, R., Bergerud, R., Bertiglia, F., Bojkovski, J., Böse, N., Brunet, M., Cappella, A., Coppa, G., del Campo, D., Dobre, M., Drnovsek, J., Ebert, V., Emardson, R., Fernicola, V., Flakiewicz, K., Gardiner, T., Garcia-Izquierdo, C., Georgin, E., Gilabert, A., Grykalowska, A., Grudniewicz, E., Heinonen, M., Holmsten, M., Hudoklin, D., Johansson, J., Kajastie, H., Kaykisizli, H., Klason, P., Knazovická, L., Lakka, A., Kowal, A., Müller, H., Musacchio, C., Nwaboh, J., Pavlasek, P., Piccato, A., Pitre, L.,de Podesta, M., 
Rasmussen, M. K., Sairanen, H., Smorgon, D., Sparasci, F., Strnad, R., Szmyrka-Grzebyk, A., and Underwood, R.: The MeteoMet project-metrology for meteorology: challenges and results, Meteorol. Appl., 22, 820-829, 2015.

Santana, M. A. A., Guimarães, P. L., Lanza, L. G., and Vuerich, E.: Metrological analysis of a gravimetric calibration system for tipping-bucket rain gauges, Meteorol. Appl., 22, 879-885, 2015.

Taylor, K. E.: Summarizing multiple aspects of model performance in a single diagram, J. Geophys. Res., 106, 7183-7192, 2001.
UNI 11452:2012: Hydrometry - Measurement of rainfall intensity (liquid precipitation) - Metrological requirements and test methods for catching type gauges, Standard, Ente Nazionale Italiano di Unificazione, Milano, IT, 2012.

WMO: Guide to Meteorological Instruments and Methods of Observation-No. 8, World Meteorological Organization, 7th edn., 2008. 